\title{
E-BAND CPW RING HYBRID USING AN OVERLAPPED COUPLING STRUCTURE
}

\author{
Jong-Sik Lim ${ }^{1}$, Sunyoung Jeong ${ }^{1}$, Young-Taek Lee ${ }^{1}$, Sungwon Kim ${ }^{1}$, Kwang-Seok Seo ${ }^{1}$, \\ and Sangwook Nam ${ }^{1}$
}

\author{
${ }^{1}$ School of Electrical Engineering and Computer Science, Seoul National University, Seoul, Rep. Of Korea \\ Applied Electromagnetics Lab., Institute of New Media and Communications, Seoul National University, \\ San 56-1, Shilim-Dong, Kwanak-Gu, Seoul, 151-742, Rep. Of Korea
}

\begin{abstract}
A novel E-band coplanar waveguide (CPW) $180^{\circ}$ ring hybrid coupler is presented. The proposed ring hybrid adopts a vertically overlapped coupling structure (OCS) having the length of $0.25 \lambda_{\mathrm{g}}$ to replace the conventional $0.75 \lambda_{\mathrm{g}}$ line or previous short-ended coupling section on the same plane. The OCS is realized by placing two metal layers to face them with each other up and down threedimensionally. The OCS permits the ring hybrid to have a small size and wideband operation due to the broadside coupling. The proposed $180^{\circ}$ ring hybrid coupler is fabricated using MMIC technology. The predicted and measured performances show that the operating frequency of the ring hybrid covers $E$ - to W-band $(60 \sim 110 \mathrm{GHz})$ with a good agreement preserved.
\end{abstract}

\section{INTRODUCTION}

Ring hybrid coupler is one of the widely used components in high frequency circuits and systems. The total length of a conventional $180^{\circ}$ ring hybrid is $1.5 \lambda_{\mathrm{g}}$ in the closed section. Fig. 1(a) and (b) show the basic structure of ring hybrid and the reduced one proposed in [1], respectively. Since the first attempt to reduce the size had been made in [1], a lot of studies have been focused on new structures to obtain more compact size and/or improved performances.

Coupled line section has additional $180^{\circ}$ of electrical length plus to $0.25 \lambda_{\mathrm{g}}$ of physical length. This property has been understood as phase inverter and used widely in design broadband, small-sized ring hybrids. A number of CPW and CPS ring hybrids were achieved by adopting coupling structures of $0.25 \lambda_{\mathrm{g}}$ and phase inverters to replace the required line section of $0.75 \lambda_{\mathrm{g}}$ [2-5].

A novel CPW ring hybrid having wideband performance and compact size is proposed in this work. A three-dimensionally (or vertically) overlapped coupling structure (OCS) with the physical length of $0.25 \lambda_{\mathrm{g}}$ is adopted instead of the conventional $0.75 \lambda_{\mathrm{g}}$ line and short-ended coupling section on the same plane.

\section{StRUCTURE OF THE PROPOSED RING HyBRID}

The coupled line section of $0.25 \lambda_{\mathrm{g}}$ shown in Fig. 1(b) is a typical phase inverter to replace the $270^{\circ}$ line. This type of coupling section has been widely implemented in microstrip or CPW ring hybrids for relatively low frequency applications because of simple structure and fabrication process. However, especially in ultra high frequency region, CPW coupled line section requires a lot of troublesome considerations in practical design and layout, because the distance between ground planes should be modified at this section. In addition, basically, the coupled line section shown in Fig. 1(b) is a bandlimited structure.

Ryu et al. proposed a MMIC 3-dB directional coupler using an air-gap stacked (AGS) microstrip lines [6], which is shown in Fig. 2(a). The physical dimensions, $\mathrm{W} 1, \mathrm{~W} 2$, and G, determine the electrical performances. The similar coupling structure named OCS is used in this work to build the proposed CPW $180^{\circ}$ ring hybrid as shown in Fig. 2(b). The broadside coupling structure results in the right electrical performances expected over wide band even at the very high frequency band.

It is possible to achieve a very broadband coupling using AGS on microstrip line. However in the microstrip case, for high frequency applications, the 
GaAs substrate should be lapped so that the final thickness may be less than $100 \mathrm{um}$ or $75 \mathrm{um}$. Additionally, W2 must be much broader than W1 for broadband coupling. However, in OCS on CPW, it does not require lapping process although the operating frequency is around 100GHz. Furthermore, the width of metal layers need not to be different nor wide because the ground planes are on the same level of the bottom metal at both sides. The physical dimensions of Fig. 2(a) in [6] are; $\mathrm{W} 1=20 \mathrm{um}, \mathrm{W} 2=40 \mathrm{um}, \mathrm{G}=2.5 \mathrm{um}$, for the operation over $23 \sim 45 \mathrm{GHz}$, while in the proposed OCS, the width of $\mathrm{W} 1(=\mathrm{W} 2)$ is only $14.6 \mathrm{um}$ and $\mathrm{G}=3 \mathrm{um}$.

Fig. 3 shows the layout and three-dimensional structure of the proposed E-band $180^{\circ} \mathrm{CPW}$ ring hybrid. The OCS exists between port 3 and 4. It is illustrated that two metal layers face with each other up and down three-dimensionally. One post is connected to the middle of the top line to support it. There is no damage in performances due to the supporting post. Two ends of coupled lines are shorted to the ground plane properly so that overlapped strip lines do not contact each other.

\section{FABRICATION AND PERFORMANCES}

The proposed E-band CPW $180^{\circ}$ ring hybrid has been built using a MMIC fabrication technology on a 650 $\mu \mathrm{m}$-thick GaAs substrate with the dielectric constant of 12.9. The thickness of lower and upper metal in the overlapped coupling section are $0.5 \mu \mathrm{m}$ and $3 \mu \mathrm{m}$, respectively. The widths of two metal lines are fixed to be the same.

Fig. 4(a) shows the predicted performance of the proposed ring hybrid. An electromagnetic simulator using method of moment has been selected in calculating S-parameters. When port 4 is the input power, as an example, the output power appears equally at port 1 and 3 while port 2 is isolated. The matching at all ports is being kept under $-10 \mathrm{~dB}$ over $60 \sim 110 \mathrm{GHz}$.

Fig. 4(b) shows the measured S-parameters of the fabricated ring hybrid. It is shown that the equally divided output power appear at port 1 and 3 even though there are minor insertion losses. The measured isolation $\left(\mathrm{S}_{24}\right)$ is excellent over the operating frequency, and the matching characteristics $\left(\mathrm{S}_{11}, \mathrm{~S}_{22}, \mathrm{~S}_{33}, \mathrm{~S}_{44}\right)$ are quite successful. The measured performances are in good agreement with the predicted S-parameters over all frequency bands.

Fig. 5 shows the measured phase difference between $\mathrm{S}_{21}$ and $\mathrm{S}_{43}$. Although some deviation is observed, the phase difference is close to $180^{\circ}$ over $60 \sim 110 \mathrm{GHz}$. It is believed that the minor phase error has been caused by the practical modification of layout for measurement, i.e. the bended port for measurement, and the measurement setup and calibration.

\section{CONCLUSION}

A new type of $\mathrm{CPW} 180^{\circ}$ ring hybrid using the overlapped coupling structure has been proposed. A GaAs MMIC fabrication technology has been used for building an example of the proposed circuit. Due to the three-dimensionally overlapped coupling structure, a very wideband operation has been achieved. The measured power division, matching, and isolation performances were excellent and in good agreement with the predicted ones over E- to W-band (60 $110 \mathrm{GHz})$.

\section{ACKNOWLEDGEMENT}

This work was supported by KOSEF under the NRL program through the Millimeter-wave Research Laboratory at Seoul National University in Korea.

\section{REFERENCES}

[1] S. March, "A Wideband Stripline Hybrid Ring," IEEE Trans. Microwave Theory Tech., vol. MTT-16, pp.361, Jun. 1968.

[2] M.-H. Murgulescu, E. Moisan, P. Legaud, E. Penard, and I. Zaquine, " New wideband, $0.67 \lambda_{\mathrm{g}}$ circumference $180^{\circ}$ hybrid ring coupler," Electronics Letters, Vol. 30, No.4, pp.299-300, Feb. 1994.

[3] C.-H. Ho, L. Fan, and K. Chang, "Broad-band uniplanar hybrid-Ring and Branch-Line Couplers," IEEE Trans. Microwave Theory Tech, Vol. 41, No.12, pp.2116-2125, Dec. 1993.

[4] C.-Y. Chang and C.-C. Yang, "A Novel Broad-Band Chebyshev-Response Rat-Race Ring Coupler," IEEE Trans. Microwave Theory Tech, Vol. 47, No.4, pp.455-462, Apr. 1999.

[5] T. Wang and K. Wu, "Size-Reduction and bandBroadening Design Technique of Uniplanar hybrid Ring Coupler Using phase Inverter for $\mathrm{M}(\mathrm{H}) \mathrm{MIC}$ 's" IEEE Trans. Microwave Theory Tech, Vol. 47, No.2, pp.198-206, Feb. 1999.

[6] G.-H. Ryu, D.-H. Kim, J.-H. Lee, and K.-S. Seo, “A Novel 3-dB Coupler for MMIC Using Air-Gap Stacked Microstrip Lines," IEEE Microwave Guide Wave Lett. Vol.10, No.1, pp.1-3, Jan. 2000. 


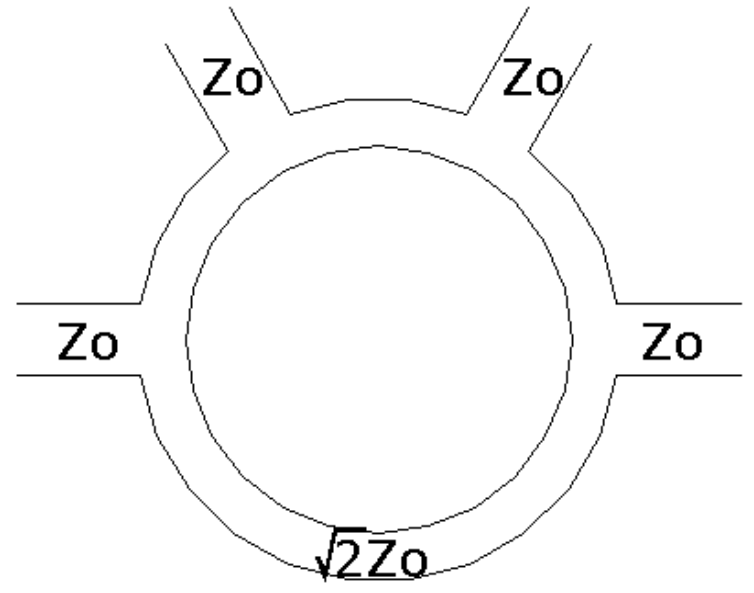

(a)

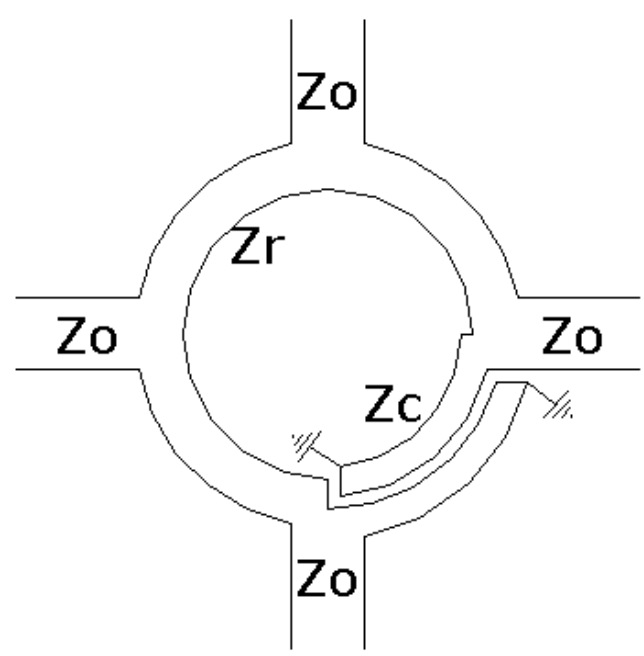

(b)

Fig. 1. (a) Basic ring hybrid (b) Reduced ring hybrid

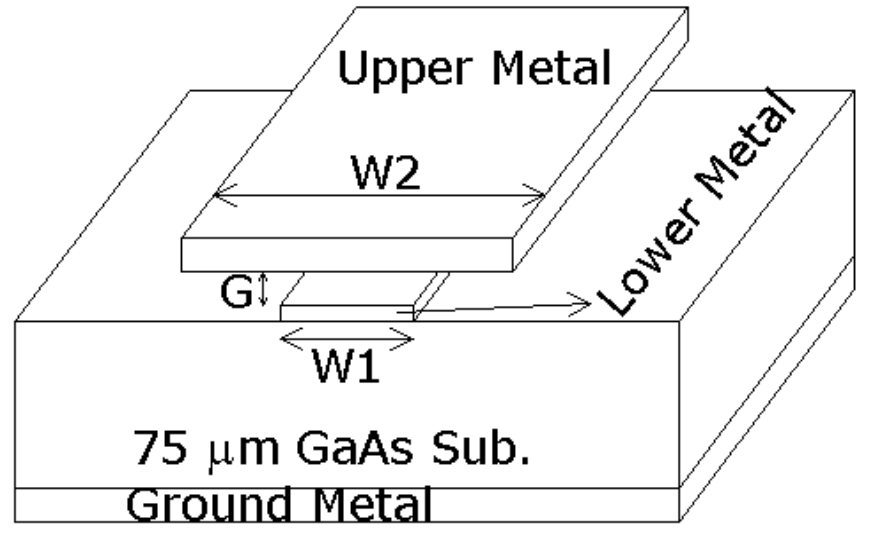

(a)

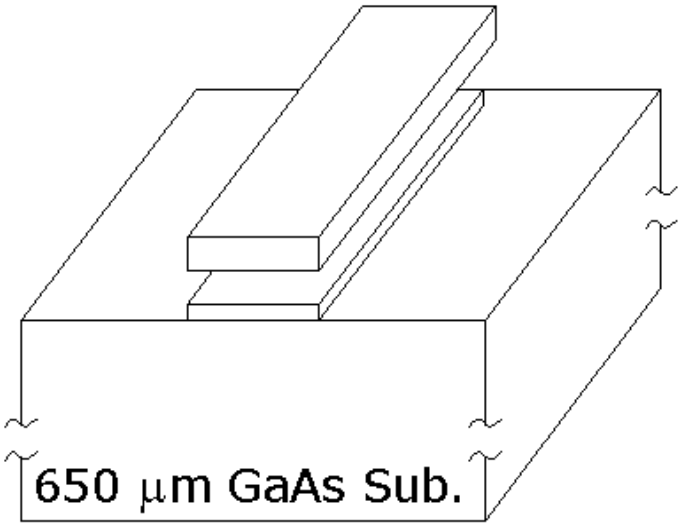

(b)

Fig. 2. (a) Air-gap stacked microstrip line proposed in [6] (b) Overlapped coupling structure on CPW
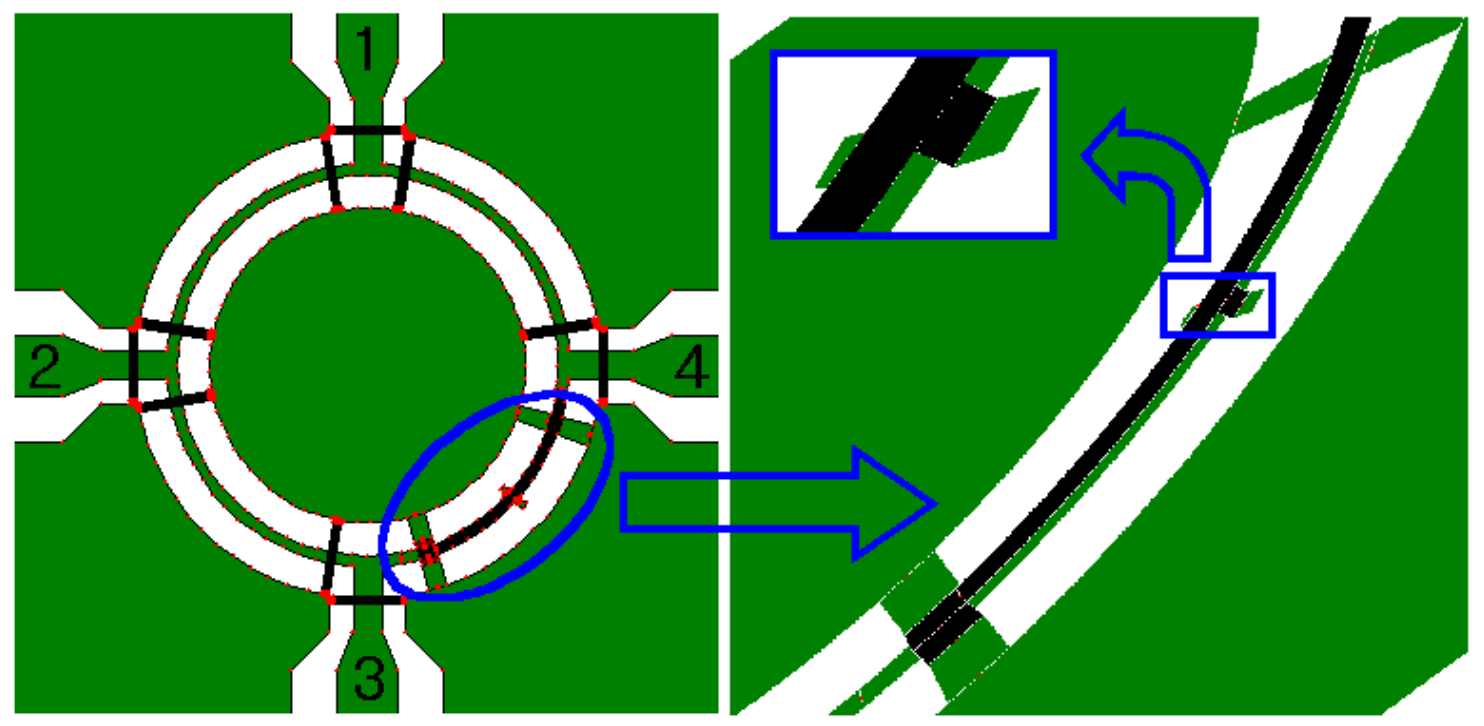

Fig. 3. CPW $180^{\circ}$ ring hybrid with the overlapped coupling structure 


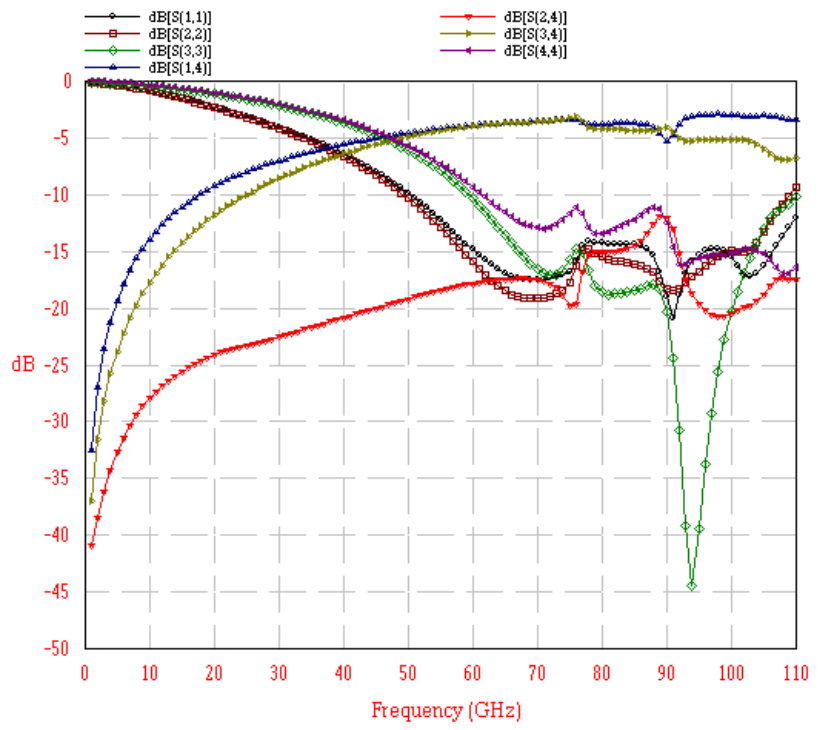

(a)

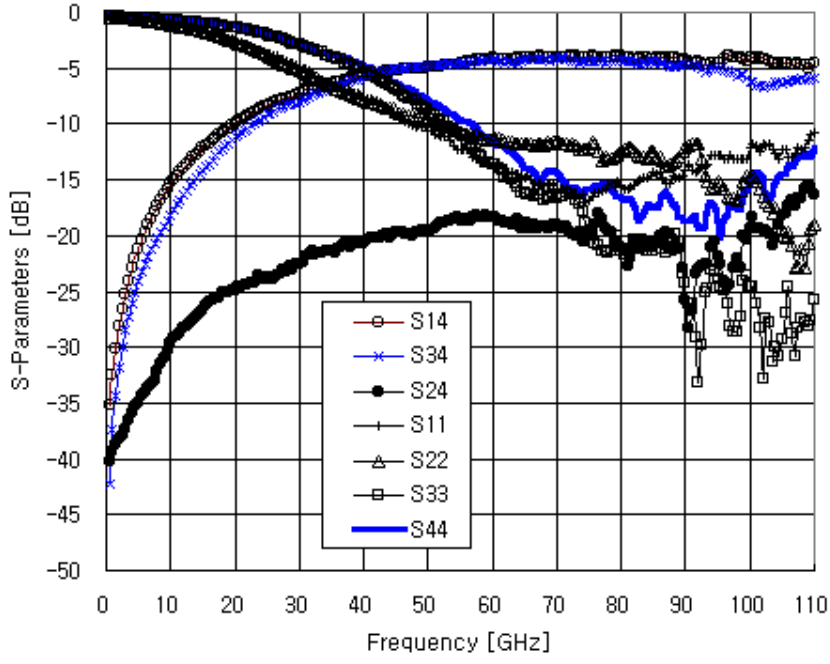

(b)

Fig. 4. Matching $\left(\mathrm{S}_{11}, \mathrm{~S}_{22}, \mathrm{~S}_{33}, \mathrm{~S}_{44}\right)$, power dividing $\left(\mathrm{S}_{14}, \mathrm{~S}_{34}\right)$ and isolation $\left(\mathrm{S}_{24}\right)$ performances (a) Simulation (b) Measurement

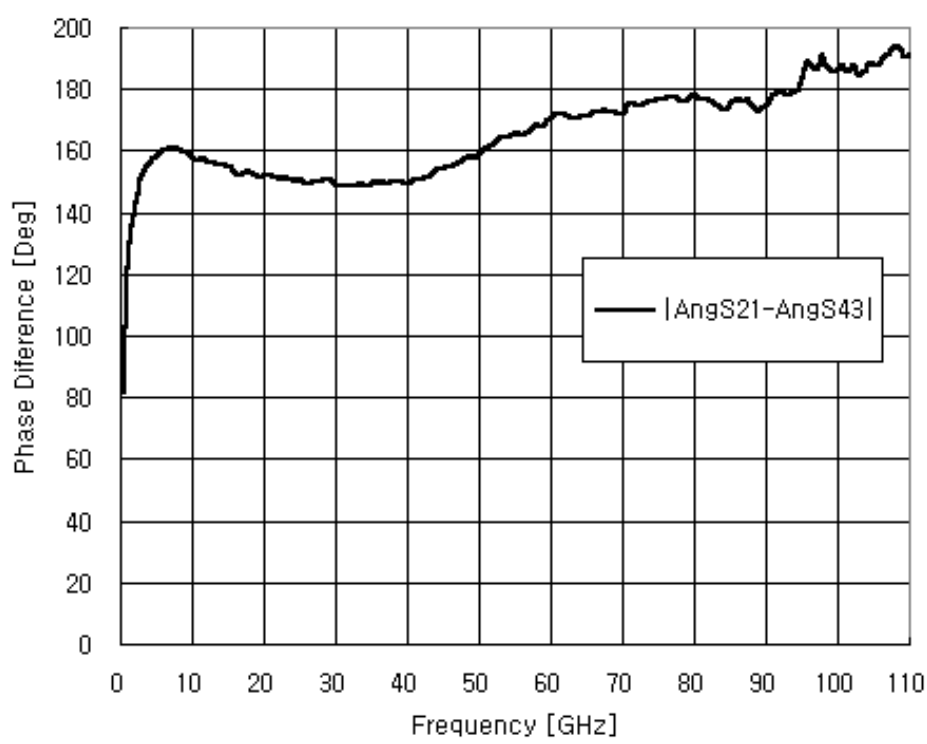

Fig. 5. Measured phase difference 